\title{
Vectra 3D Imaging for Quantitative Volumetric Analysis of the Efficacy of Propranolol in Infantile Hemangioma
}

\author{
Reina Kitabata1, Noriko Aramaki-Hattori'1, Hiroyuki Fukushima², Jun Maeda², Tomoki Kiuchi ${ }^{3}$, \\ Marie Aoki ${ }^{4}$, Kazuo Kishi ${ }^{1}$ \\ ${ }^{1}$ Department of Plastic and Reconstructive Surgery, Keio University School of Medicine, Tokyo, Japan \\ ${ }^{2}$ Department of Pediatrics, Keio University School of Medicine, Tokyo, Japan \\ ${ }^{3}$ Department of Plastic and Reconstructive Surgery, International University of Health and Welfare Hospital, Nasushiobara, \\ Tokyo, Japan \\ ${ }^{4}$ Department of Plastic and Reconstructive Surgery, Tokyo Metropolitan Children's Medical Center, Tokyo, Japan \\ Email: nonken@2001.jukuin.keio.ac.jp
}

How to cite this paper: Kitabata, R., Aramaki-Hattori, N., Fukushima, H., Maeda, J., Kiuchi, T., Aoki, M. and Kishi, K. (2019) Vectra 3D Imaging for Quantitative Volumetric Analysis of the Efficacy of Propranolol in Infantile Hemangioma. Modern Plastic Surgery, 9, 45-51.

https://doi.org/10.4236/mps.2019.93006

Received: March 26, 2019

Accepted: July 28, 2019

Published: July 31, 2019

Copyright (c) 2019 by author(s) and Scientific Research Publishing Inc. This work is licensed under the Creative Commons Attribution International License (CC BY 4.0).

http://creativecommons.org/licenses/by/4.0/

\begin{abstract}
Infantile hemangioma (IH) is a common tumor in infants. After the proliferative phase, hemangiomas regress, but sometimes leave scars. Propranolol is now the recommended first-line oral therapy for IH. To evaluate the effectiveness of oral propranolol administration, we measured quantitative changes in an IH by digital camera and Computed Tomography (CT) or Magnetic Resonance Imaging (MRI). Although digital cameras are very simple, changes in color tones occur with time, lesions are evaluated in two dimensions, and changes in the thickness of the hemangioma are difficult to evaluate. Therefore, we investigated the feasibility of $3 \mathrm{D}$ photography to quantify volumes in IH during oral propranolol treatment.
\end{abstract}

\section{Keywords}

Infantile Hemangioma, Propranolol, 3D Photography

\section{Introduction}

Infantile hemangiomas (IHs) rapidly increase in size from birth until 5 months of age [1]. After the proliferative phase, the color of most IHs fades and the size decreases. Because it takes a long time for the color of IHs to fade and residual lesions have a wrinkle-like appearance, it is sometimes necessary to surgically remove scars. The effectiveness of oral propranolol in the treatment of IHs was first reported in Europe in 2008 [2], and a global randomized control study was 
conducted [3] [4]. Propranolol is now the recommended first-line oral therapy for IH. The oral administration of propranolol for treatment of $\mathrm{IH}$ has been covered by national insurance in Japan since 2017. Before national insurance approval, we offered this treatment in our hospital after review and approved by the Ethical Review Board of Keio University School of Medicine (approval code No. 20130027).

To evaluate the effectiveness of propranolol oral administration, we first measured quantitative changes in IHs by digital camera and CT or MRI. Although digital cameras are very simple, changes in color tones occur over time, lesions are evaluated in two dimensions, and changes in the thickness of IHs are difficult to evaluate. Because the indication was limited by expense and radiation exposure, there were many patients with superficial lesions who were ineligible, but there were several patients who were eligible, such as those with an $\mathrm{IH}$ in the orbital space or huge and deep IHs. To overcome the shortcomings, we used a 3D camera (Vectra $\mathrm{H} 1$; Canfield Scientific, Inc., NJ, USA) to measure quantitative changes in IHs. In this study, we determined whether or not 3D photography is a useful tool for measuring the surface area and volume of IHs over time during treatment.

\section{Patients and Methods}

This study included 10 patients treated with oral propranolol treatment from 2015-2016 in our hospital before propranolol treatment was covered by insurance in Japan. All IHs were in the proliferative phase and 3 months after. Oral propranolol treatment was provided for those who were expected to cause serious dysfunction such as respiratory and visual function or expected to have surgical resection after spontaneous regression, and for IHs in areas likely to be wedge-shaped (nose, lips, auricle, intercostal, etc.). The patients signed informed consult. Baseline screening by pediatric cardiologists included blood pressure, heart rate, hematologic examination, an echocardiogram, and an electrocardiogram. We initiated propranolol treatment in the hospital at a dose of $1 \mathrm{mg} / \mathrm{kg}$ per day and increased the dose to $2 \mathrm{mg} / \mathrm{kg}$ per day. After discharge from the hospital, we followed the patients in the outpatient clinic. We slowly tapered the $2 \mathrm{mg} / \mathrm{kg}$ per day dose after 6 months and stop the medication. 3D photographs of the IHs were taken using a 3D camera (Vectra ${ }^{\circ} \mathrm{H}$ ) before starting the medication, and 1, 3, and 6 months after starting the medication). The Vectra $\mathrm{H} 1$ is a handy $3 \mathrm{D}$ camera system; the images are easy to analyze and the volume measurements are easy to obtain. Because the $3 \mathrm{D}$ camera system is designed to take pictures of people, the shooting time is 0.002 seconds, the color image is highly accurate, and background noise is suppressed. 3D reconstruction was generated using the attachment software for the $3 \mathrm{D}$ camera. The $\mathrm{IH}$ lesion outlines were manually traced to automatically calculate the surface area and volume.

\section{Ethical Considerations}

This study was reviewed and approved by the Ethical Review Board of Keio 
University before patient enrollment and data collection (approval code No. 20130027). The purpose of the study was explained in detail and the participants' parents voluntarily submitted a signed, written informed consent. Careful consideration was paid to protecting the privacy and personal information of the subjects.

\section{Results}

The average age at starting medication was 3.5 months. The study cohort consisted of 2 boys and 8 girls (Table 1). We calculated the IH surface area and volume at the start of treatment, and 1, 3, and 6 months after starting medication and followed up until three years old (1 case was lost to follow-up 1 month after starting medication and 2 cases were lost to follow-up 3 months after starting medication). The complication of treatments (hypotension, bradycardia, hypoglycemia, hyperkalemia, wheezing) does not occur. Case 1 was a 5-month-old boy. Two weeks after birth he developed an IH on the right chest and was referred to our hospital for oral treatment. Oral medication with a $\beta$--blocker was initiated at $2 \mathrm{mg} / \mathrm{kg} /$ day according to our protocol. 3D photographs of the $\mathrm{IH}$ were obtained before starting medication, and 1,3, and 6 months after starting medication using a 3D camera (Vectra $\mathrm{H} 1$ ). A 3D image before starting medication is shown in Figure 1. By 6 months after starting medication, the color of the IH had faded, but it was difficult to determine the surface area and volume of the IH using a 2D digital camera (Figure 2(a) and Figure 2(b)). We simulated the chest wall using $3 \mathrm{D}$ photography from the $3 \mathrm{D}$ coordinates of the $\mathrm{IH}$ margin and calculated the volume of the IH (Figure 3(a)). The volume was $23.3 \mathrm{cc}$. Six months after starting oral administration, the volume of the IH was $5.4 \mathrm{cc}$ (Figure 3(b)). We also calculated the change in IH over time using the following formula: IH volume 1, 3, or 6 months after treatment (cc)/original IH volume $(\mathrm{cc}) \times 100(\%)$. The volume of the IH lesion was significantly decreased during treatment $(40.86 \% \pm 17.19 \%$ at 1 month, $25.06 \% \pm 14.41 \%$ at 3 months, and $8.69 \% \pm 7.29 \%$ at 6 months; $p<0.001$; Figure 4$)$. The surface area of the IH was significantly decreased during treatment $(82.23 \% \pm 13.68 \%$ at 1 month, $\mathrm{p}<0.01$; $78.09 \% \pm 28.79 \%$ at 3 months, $\mathrm{p}<0.05$; and $64.72 \% \pm 22.36 \%$ at 6 months, $\mathrm{p}<$ 0.001 ; Figure 4). Case 2 is a 3 -month-old girl. The change in IH over time is shown in Figure 5.

\section{Discussion}

A $\beta$-blocker has fewer side effects in treating IHs compared to steroids and interferon; especially good results have been reported in IHs involving the head and neck [5]. Although the mechanism of action of propranolol in treating IHs is not clear, propranolol is a non-selective beta-adrenergic antagonist with an affinity for $\beta$-1 and $\beta$-2 receptors. Propanolol causes down-regulation of bFGF and VEGF and induces apoptosis of capillary endothelial cells [6]. It has also been reported that propanolol selectively inhibits MMP-9 [6]. Propranolol has effica- 
cy in the treatment of proliferating IH and facilitating the involution phase early, thus possibly avoiding surgery.

Using a 3D camera (Vectra $\mathrm{H} 1$ ) is as easy as using a digital camera. Indeed, a $3 \mathrm{D}$ camera can easily measure the volume of IH lesions. 3D measurement is non-invasive, does not require radiation exposure and sedation as do CT and MRI, and is very useful in evaluating the treatment effect in infants. The reliability of 3D photography for IH volume changes over time (3 months after taking medication) has been reported [7]. Our study showed the volume and surface area changes in IHs by 6 months, as determined by 3D imaging. We should also consider the surface area. In case 3, a 4-month-old girl, even though no change in surface area was demonstrated, a decrease in volume was observed (Figure 6). It is our opinion that quantitative evaluation of IHs using a 3D camera is useful.

Table 1. Data of 2 boys and 8 girls.

\begin{tabular}{ccccccc}
\hline No. & Location & Age & Sex & Weight & Recurrence & Adverse Event \\
\hline 1 & Chest & $6 \mathrm{~m}$ & M & 7.2 & - & - \\
2 & Jaw & $3 \mathrm{~m}$ & $\mathrm{~F}$ & 5.4 & - & - \\
3 & Cheek & $3 \mathrm{~m}$ & $\mathrm{~F}$ & 6.9 & - & - \\
4 & Lip & $3 \mathrm{~m}$ & $\mathrm{M}$ & 5.9 & - & - \\
5 & Eyelid & $3 \mathrm{~m}$ & $\mathrm{~F}$ & 6.1 & - & - \\
6 & Eyelid & $4 \mathrm{~m}$ & $\mathrm{~F}$ & 5.3 & - & - \\
7 & Eyelid & $3 \mathrm{~m}$ & $\mathrm{~F}$ & 5.5 & - & - \\
8 & Nose & $3 \mathrm{~m}$ & $\mathrm{~F}$ & 5.7 & - & - \\
9 & Scalp & $3 \mathrm{~m}$ & $\mathrm{~F}$ & 6.6 & - & - \\
10 & Eyelid & $4 \mathrm{~m}$ & $\mathrm{~F}$ & 6.4 & - & - \\
\hline
\end{tabular}

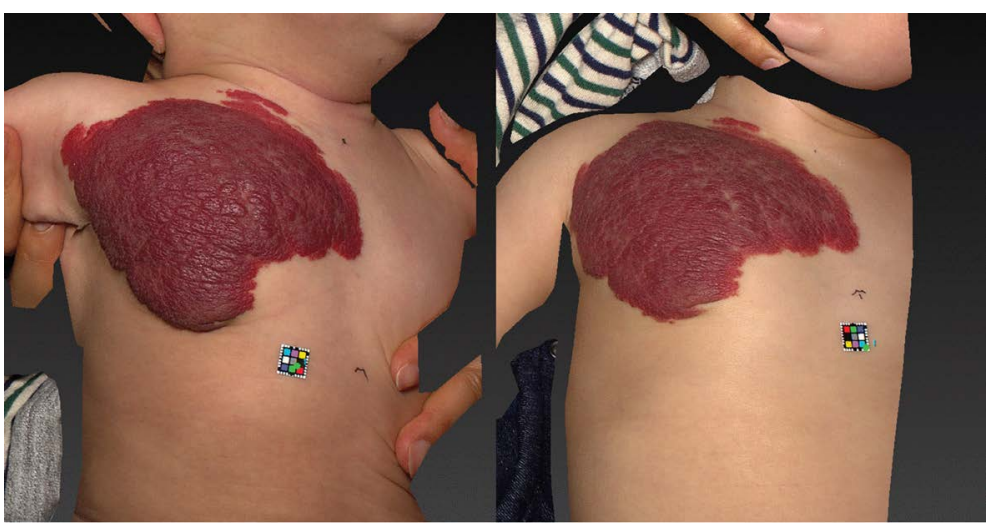

Figure 1. 3D imaging of IH in a 5-month-old boy (movie). The chest wall is simulated from the $3 \mathrm{D}$ coordinates of the hemangioma margin, and the volume of the hemangioma present on it is calculated. 


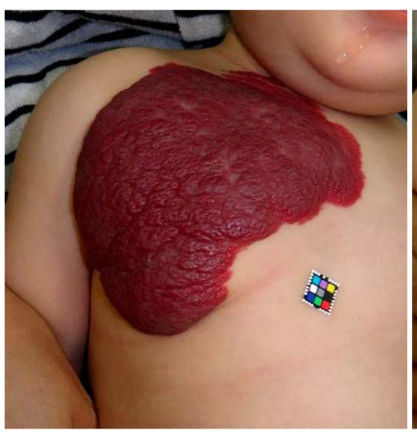

(a)

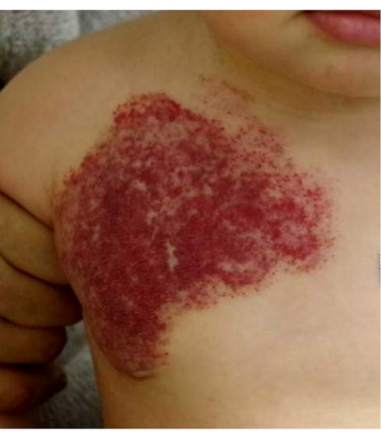

(b)

Figure 2. Photographs by 2D camera of a 5-month-old boy. (a) before starting medication; (b) after 6 months of propranolol treatment. Although these images show the change of flattens and fades, it is difficult to quantify the change in volume.

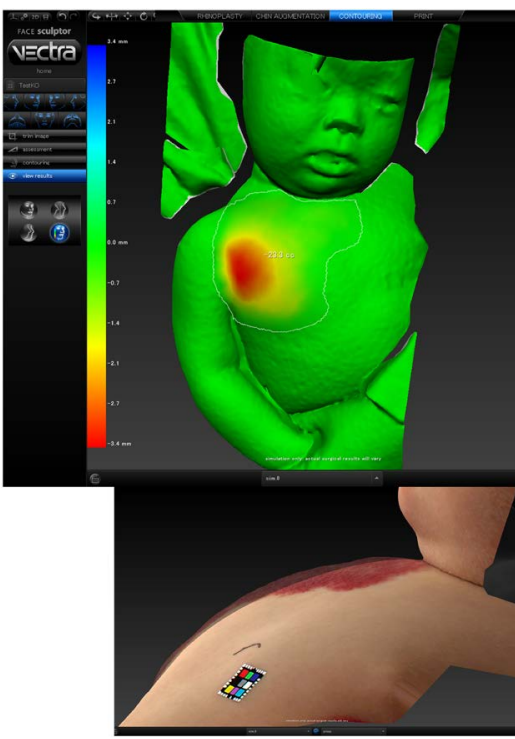

(a)

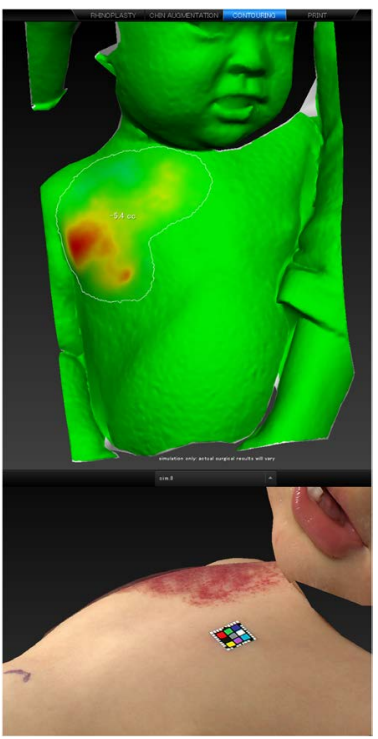

(b)

Figure 3. Volumetric measurement using 3D photography of an $\mathrm{IH}$ in a 5-month-old boy. (a) before starting medication; Volume: $23.3 \mathrm{cc}$, Height difference between chest wall and hemangioma: 6 - $10 \mathrm{~mm}$. (b) after 6 months of propranolol treatment. Volume: $5.4 \mathrm{cc}$, Height difference between chest wall and hemangioma: 2 - $3.5 \mathrm{~mm}$.

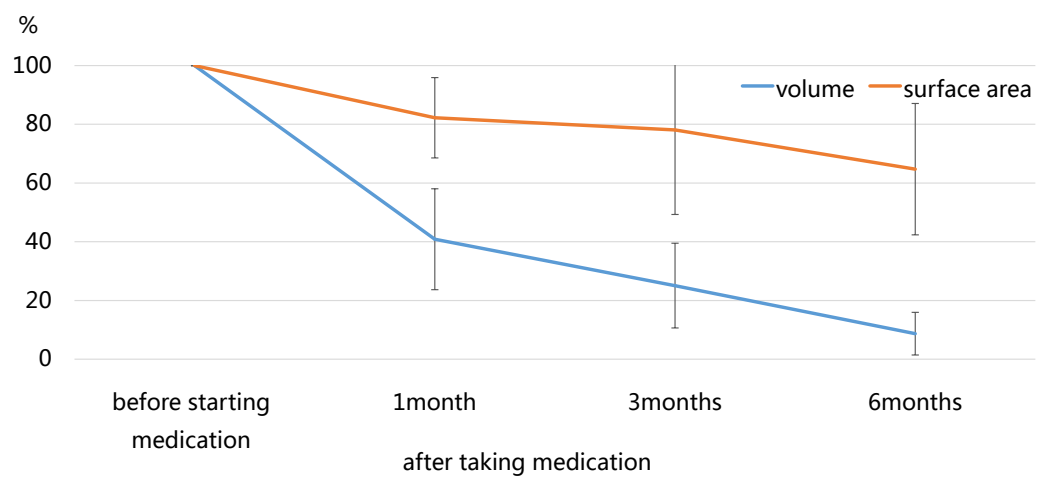

Figure 4. Volume and surface area change of IH during treatment. It shows the usefulness of the evaluation with a $3 \mathrm{D}$ camera. 


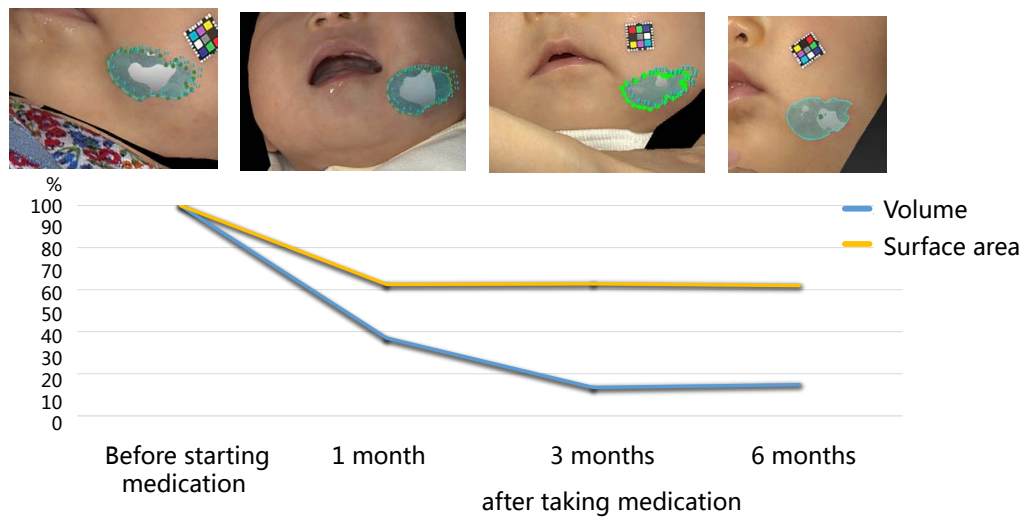

Figure 5. Changes in volume and surface area of $\mathrm{IH}$ during treatment in a 3 month-old girl. Although the surface area has not decreased, the volume has decreased by $80 \%$. It shows the usefulness of the evaluation with a $3 \mathrm{D}$ camera.

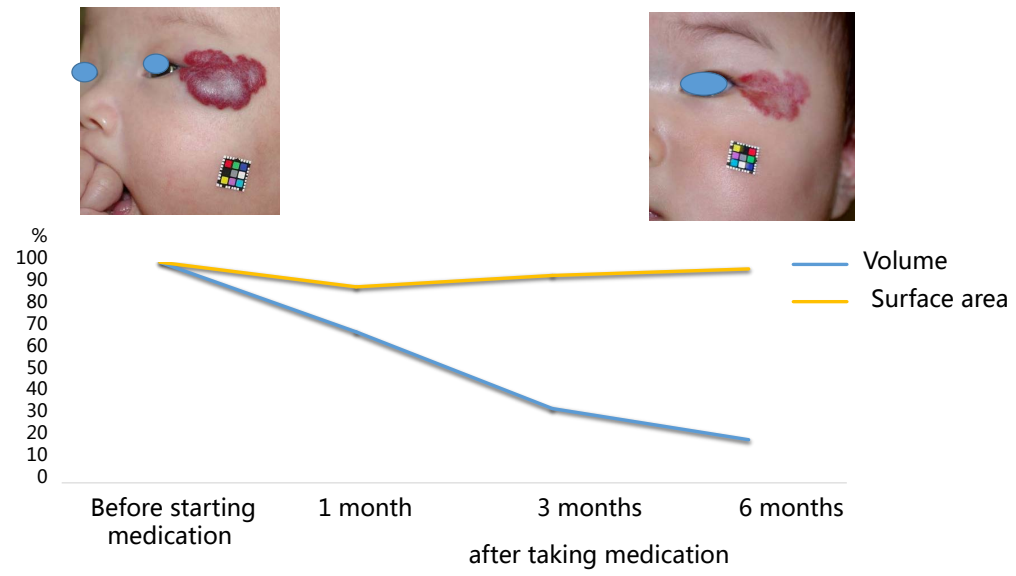

Figure 6. Changes in volume and surface area of IH during treatment in a 4-month-old girl. It shows that the surface area does not change much, but the volume is clearly reduced and improved with a $3 \mathrm{D}$ camera.

In this study, we followed infants for 6 months by using a 3D camera to measure the surface area and volume of IHs. The IH volume was shown to be greatly reduced with propranolol treatment compared with baseline. This result showed the usefulness of evaluation with a 3D camera. Even in cases in which the surface area did not change, the volume clearly decreased. A drawback of 3D imaging is that the shape of the lesion and simulation of the normal form may change and the camera is expensive. Nevertheless, we suggest that volumetric measurement of IHs using a 3D camera may be useful to evaluate treatment.

\section{Conflicts of Interest}

The authors declare no conflicts of interest regarding the publication of this paper.

\section{References}

[1] Chang, L.C., Haggstrom, A.N., Drolet, B.A., et al. (2008) Growth Characteristics of 
Infantile Hemangiomas: Implications for Management. Pediatrics, 122, 360-367.

[2] Léauté-Labrèze, C., Dumas de la Roque, E., Hubiche, F., et al. (2008) Propranolol for Severe Hemangiomas of Infancy. The New England Journal of Medicine, 358, 2649-2651.

[3] Bauman, N.M., McCarter, R.J., Guzzetta, P.C., et al. (2014) Propranolol vs Prednisolone for Symptomatic Proliferating Infantile Hemangiomas: A Randomized Clinical Trial. JAMA Otolaryngology_Head \& Neck Surgery, 140, 323-330. https://doi.org/10.1001/jamaoto.2013.6723

[4] Léauté-Labrèze, C., Hoeger, P., Mazereeuw-Hautier, J., et al. (2015) A Randomized, Controlled Trial of Oral Propranolol in Infantile Hemangioma. The New England Journal of Medicine, 372, 735-746.

[5] Fuchsmann, C., Quintal, M.C., Giguere, C., et al. (2011) Propranolol as First-Line Treatment of Head and Neck Hemangiomas. Archives of Otolaryngology_Head \& Neck Surgery, 137, 471-478. https://doi.org/10.1001/archoto.2011.55

[6] Léauté-Labrèze, C. and Taieb, A. (2008) Efficacy of $\beta$-Blockers in Infantile Haemangiomas: The Physiopathological Significance and Therapeutic Consequences. Annales de Dermatologie et de Vénéréologie, 135, 860-862. https://doi.org/10.1016/j.annder.2008.10.006

[7] Robertson, S., Kimble, R., Storey, K., Gee Kee, E.L. and Stockton, K.A. (2016) 3D photography Is a Reliable Method of Measuring Infantile Haemangioma Volume Over Time. Journal of Pediatric Surgery, 51, 1552-1556.

https://doi.org/10.1016/j.jpedsurg.2016.04.013 